14,07

\title{
Механизмы роста и структура кластеров 2D-нанонаполнителя в полимерных средах
}

\author{
(C) Г.В. Козлов, И.В. Долбин ॠ \\ Кабардино-Балкарский государственный университет им. Х.М. Бербекова \\ Нальчик, Россия \\ ฯ E-mail: i_dolbin@mail.ru \\ (Поступила в Редакцию 5 мая 2018 г.)
}

\begin{abstract}
Оценки в рамках фрактального анализа продемонстрировали, что пластины 2D-нанонаполнителя в полимерных средах формируют кластеры (тактоиды), которые являются фрактальными объектами. Этот вывод подтвержден с использованием модели необратимой агрегации. Основным фактором, определяющим размерность указанных кластеров, является размерность случайного блуждания исходных наночастиц. Структура тактоидов 2D-нанонаполнителя однозначно определяет модуль упругости нанокомпозита при фиксированном содержании нанонаполнителя.
\end{abstract}

DOI: $10.21883 /$ FTT.2019.01.46910.134

\section{1. Введение}

Как известно [1], изначально полимерные нанокомпозиты, в том числе и производимые в промышленном масштабе, были получены с использованием 2D-нанонаполнителей, а именно, органоглин разных видов. В последнее десятилетие появился ряд новых 2D-нанонаполнителей (графен [2], оксид графена [3], нитрид бора [4] и т.п.), которые обнаружили высокую эффективность в изменении свойств полимерных наноматериалов. Так, введение больших количеств эсфолиированного графена (порядка 0.4-0.6) позволило увеличить модуль упругости исходного полиуретана примерно в 150 раз [5]. Объяснение этого очень сильного эффекта было дано авторами [6] в рамках перколяционных моделей „муравья“ и „термита“ при условии трактовки нанокомпозитов полиуретан/графен как случайных двухкомпонентных материалов [7].

В настоящее время хорошо известно [8], что основным эффектом при наполнении полимеров наночастицами является сильная агрегация последних, которая существенно влияет на свойства конечных наноматериалов. Для 2D-нанонаполнителей агрегация выражена формированием их пачек (тактоидов), состоящих из конечного числа исходных коллинеарных пластин [9]. Авторы [10] с помощью современных экспериментальных методик продемонстрировали, что тактоид органоглины представляет собой фрактальный объект. В работе [11] было сделано предположение, что структура нанонаполнителя, формируемая в полимерной матрице, определяет свойства нанокомпозитов. Однако никаких количественных моделей или оценок для подтверждения корректности этого предположения авторы [11] не представили. Поэтому целью настоящей работы является количественное описание механизма формирования кластеров (тактоидов) 2D-нанонаполнителей в полимерных средах и характеризация их конечной структуры в рамках фрактального анализа и моделей необратимой агрегации, а также влияния указанной структуры на свойства полученных наноматериалов с применением перколяционной модели усиления на примере нанокомпозитов поливиниловый спирт/графен [12].

\section{2. Результаты и обсуждение}

В настоящей работе выполнен теоретический анализ экспериментальных данных, полученных для нанокомпозитов поливиниловый спирт/оксид графена в работе [12]. Пластины оксида графена (ОГ) получены из порошка природного графита по методу Хаммера [12]. Сначала порошок графита очищался диализом для удаления остаточных металлических примесей, затем фильтровался и сушился под вакуумом при температуре $333 \mathrm{~K}$ в течение 24 часов. Полученный порошкообразный ОГ обрабатывался в деионизированной воде ультразвуком для образования гомогенной дисперсии [12].

В качестве матричного полимера использован поливиниловый спирт (ПВС) с молекулярной массой повторяющегося звена 2400-2500 g/mol марки 122 со степенью гидролиза 98-99\% производства фирмы Beijing Chem. (Китай). Пленки нанокомпозитов ПВС/ОГ с содержанием ОГ 1-5 mass.\% получены вакуумной фильтрацией дисперсий ПВС и ОГ в деионизированной воде через поликарбонатные мембраны с размером пор $0.2 \mu \mathrm{m}$ производства фирмы Nucleapore (Великобритания) и подвергались сушке при указанных выше условиях. Перед испытаниями пленки нанокомпозитов ПВС/ОГ дополнительно сушились при $333 \mathrm{~K}$ в течение часа [12].

Механические испытания выполнены на машине для растяжения модели WDW 3020 Autograph производства фирмы Changchun Xinke Co. (Китай). В качестве образцов использованы пленки толщиной 78-120 $\mu \mathrm{m}$ с шириной 2-3 mm и длиной 20-25 mm. Испытания выполнены при температуре $293 \mathrm{~K}$, скорости ползуна $1 \mathrm{~mm} / \mathrm{min}$ и предварительной нагрузке $1 \mathrm{~N}$ [12]. 
Авторы [13] предложили следующее уравнение для расчета фрактальной размерности $D$ объекта, полученного в результате взаимодействия двух фрактальных объектов с размерностями $D_{1}$ и $D_{2}$ соответственно

$$
D=\frac{d\left(2 D_{1}-D_{2}\right)}{d+2\left(D_{1}-D_{2}\right)},
$$

где $d$ - размерность евклидова пространства, в котором рассматривается фрактал (очевидно, в нашем случае $d=3)$.

Применительно к рассматриваемым наноматериалам размерность $D$ является соответствующей размерностью макромолекулярного клубка полимерной матрицы нанокомпозита, $D_{1}$ - размерностью такого же клубка матричного полимера и $D_{2}-$ размерностью частицы (агрегата частиц) нанонаполнителя.

Величины $D$ и $D_{1}$ можно определить согласно следующему уравнению для случая линейных полимеров [1]

$$
D\left(D_{1}\right)=\frac{d_{f}\left(d_{f 1}\right)}{1.5},
$$

где $d_{f}$ и $d_{f 1}$ - размерности структуры нанокомпозита и матричного полимера, соответственно, которые определяются согласно следующей формуле [8]:

$$
d_{f}\left(d_{f 1}\right)=(d-1)\left(1+v\left(v_{1}\right)\right)
$$

где $v$ и $v_{1}-$ коэффициент Пуассона для нанокомпозита и матричного полимера, соответственно, оцениваемый по результатам механических испытаний с помощью соотношения [14]

$$
\frac{\sigma_{\gamma}}{E}=\frac{1-2 v}{6(1+v)}
$$

где $\sigma_{\gamma}$ и $E-$ предел текучести и модуль упругости полимерного материала, соответственно.

Расчет размерности агрегатов (тактоидов) пластин оксида графена $D_{2}$ дал интервал 1.75-2.13 для диапазона массового содержания нанонаполнителя 1-5 mass.\% (рис. 1).

В работе [15] был предложен еще один метод оценки фрактальной размерности $D_{2}$ агрегата частиц нанонаполнителя, использующий следующее уравнение:

$$
L_{p l}=15 \varphi^{-1 /\left(d-D_{2}\right)},
$$

где $L_{p l}$ - длина пластины 2D-нанонаполнителя, равная в настоящем случае $800 \mathrm{~nm}$ [12], $\varphi_{n}$ - объемное содержание нанонаполнителя, определяемое следующим образом [9]:

$$
\varphi_{n}=\frac{W_{n}}{\rho_{n}}
$$

где $W_{n}$ - массовое содержание нанонаполнителя, $\rho_{n}-$ его плотность, равная для ОГ $1600 \mathrm{~kg} / \mathrm{m}^{3}$ [12].

В таблице приведено сравнение размерностей $D_{2}$, рассчитанных согласно уравнениям (1) и (5), которое

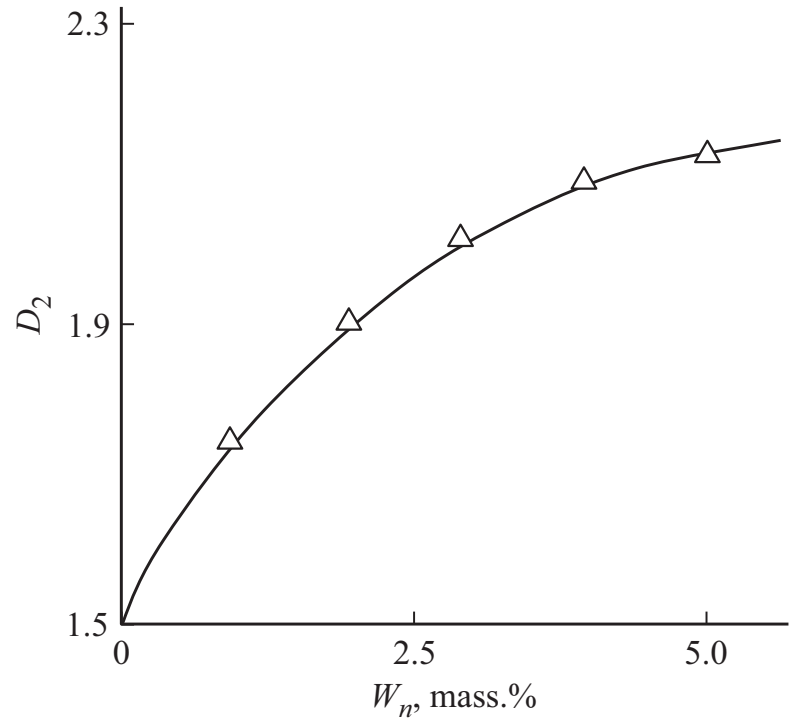

Рис. 1. Зависимость фрактальной размерности $D_{2}$ кластеров (тактоидов) оксида графена от массового содержания нанонаполнителя $W_{n}$ для нанокомпозитов ПВС/ОГ.

показало хорошее соответствие обоих наборов данных. Указанное соответствие и сравнение уравнений (1) и (5) позволяют сделать три важных вывода. Во-первых, кластеры (тактоиды) оксида графена формируются механизмом роста на множественных местах зарождения [16], что соответствует самой общей картине образования структуры нанокомпозита [8]. Это означает, что любая отдельная пластина оксида графена может стать зародышем для последующего роста тактоида. Во-вторых, из уравнения (5) при условии $L_{p l}=$ const и $d=3=$ const следует, что единственным фактором, контролирующим изменение размерности тактоидов ОГ, является вариация содержания 2D-нанонаполнителя $\varphi_{n}$. И, в третьих, из данных таблицы следует, что повышение $\varphi_{n}$ приводит к изменению механизма роста кластеров (тактоидов) ОГ от диффузионно-ограниченного $\left(D_{2} \approx 1.7\right)$ до химически-лимитированного $\left(D_{2} \approx 2.1\right)$ [17].

Рассмотрим физические основы повышения размерности тактоидов ОГ $D_{2}$ по мере роста его содержания $\varphi_{n}$. Очевидно, что увеличение $\varphi_{n}$ или числа частиц 2D-нанонаполнителя приводит к снижению времени ожидания контакта между частицами и повышению

Сравнение рассчитанных разными методами размерностей кластеров (тактоидов) оксида графена $D_{2}$

\begin{tabular}{c|c|c}
\hline $\begin{array}{c}\text { Объемное содержание } \\
\text { оксида графена, } \varphi_{n}\end{array}$ & $\begin{array}{c}D_{2}, \\
\text { уравнение (1) }\end{array}$ & $\begin{array}{c}D_{2}, \\
\text { уравнение (5) }\end{array}$ \\
\hline 0.00625 & 1.75 & 1.72 \\
0.0125 & 1.91 & 1.90 \\
0.0188 & 2.02 & 2.0 \\
0.0250 & 2.10 & 2.07 \\
0.0313 & 2.13 & 2.13
\end{tabular}


степени связности структуры, которую можно охарактеризовать эффективной спектральной размерностью $d_{s}^{\prime}$, определяемой согласно уравнению [18]:

$$
d_{s}^{\prime}=\frac{2\left(3 D_{2}-d\right)}{d+2}
$$

что позволяет выполнить оценку размерности блуждания частиц нанонаполнителя $d_{w}$ следующим простым способом [18]:

$$
d_{w}=\frac{2 d}{d_{s}} .
$$

На рис. 2 приведена зависимость $d_{w}\left(\varphi_{n}\right)$ для рассматриваемых нанокомпозитов ПВС/ОГ, которая показала резкое снижение $d_{w}$ или спрямление траекторий блуждания наночастиц ОГ по мере роста их содержания $\varphi_{n}$. Обращает на себя внимание изменение градиента зависимости $d_{w}\left(\varphi_{n}\right)$ при $\varphi_{n} \approx 0.014$, что может быть обусловлено двумя структурными факторами. Во-первых, порог перколяции $\varphi_{c}$ анизотропных частиц наиболее просто определяется согласно уравнению [19]

$$
\varphi_{c}=0.6\left(\frac{t_{p l}}{L_{p l}}\right),
$$

где $t_{p l}$ - толщина тактоида ОГ.

Авторы [12] определили величину $L_{p l} / t_{p l}$ как равную 60.8 , что согласно уравнению $(9)$ дает $\varphi_{c} \approx 0.01$. Из графика рис. 2 следует, что величина $\varphi_{c}$ хорошо согласуется со значением $\varphi_{n}$, соответствующим точке изменения градиента зависимости $d_{w}\left(\varphi_{n}\right)$. Во-вторых, как отмечалось выше, при $\varphi_{n} \approx 0.015$ реализуется изменение механизма формирования кластеров (тактоидов) пластин ОГ от диффузионно-ограниченного к химически- лимитированному. Сравнение этих двух наблюдений демонстрирует,

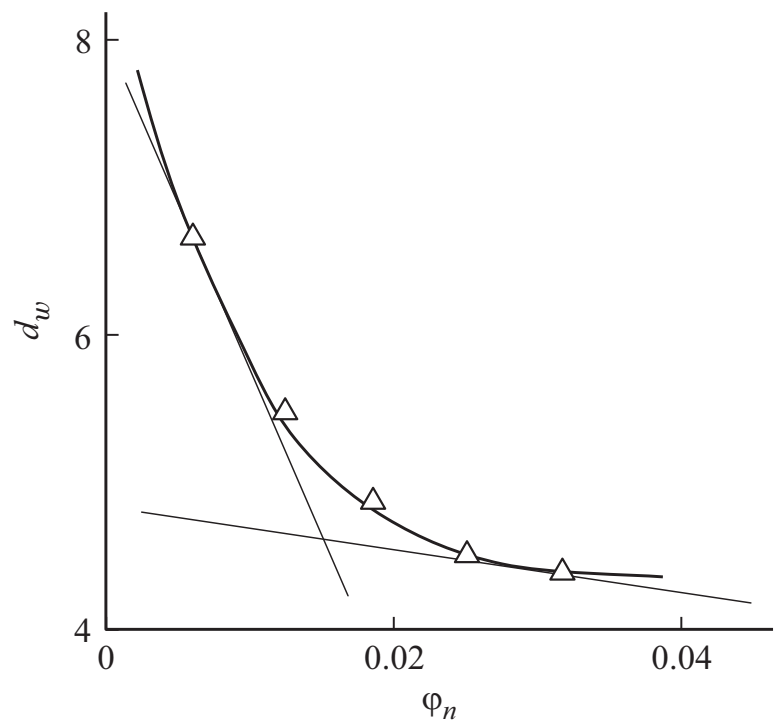

Pис. 2. Зависимость размерности блуждания наночастиц ОГ $d_{w}$ от объемного содержания нанонаполнителя $\varphi_{n}$ для нанокомпозитов ПВС/ОГ. Тонкие линии показывают касательные к разным участкам зависимости $d_{w}\left(\varphi_{n}\right)$.

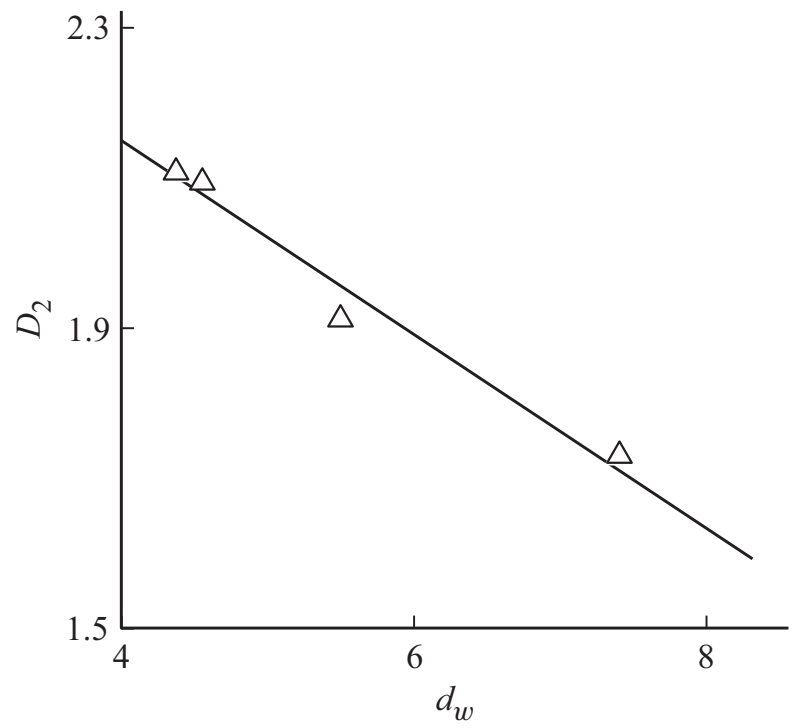

Рис. 3. Зависимость фрактальной размерности кластеров (тактоидов) оксида графена $D_{2}$ от размерности блуждания частиц 2D-нанонаполнителя $d_{w}$ для нанокомпозитов ПВС/ОГ.

что при $\varphi_{n}<\varphi_{c}$ реализуется первый из указанных механизмов, а при $\varphi_{n} \geqslant \varphi_{c}-$ второй, т.е. реализация того или иного механизма формирования тактоидов также определяется структурными факторами.

Как известно [13], величина размерности $D_{2}$ кластера ОГ является функцией размерности блуждания $d_{w}$ пластин ОГ, его составляющих - чем меньше $d_{w}$, тем больше $D_{2}$. На рис. 3 приведена зависимость $D_{2}\left(d_{w}\right)$, которая показала ожидаемый рост $D_{2}$ по мере снижения $d_{w}$ (обусловленного увеличением $\varphi_{n}$, рис. 2 ), что аналитически можно выразить следующим образом:

$$
D_{2}=2.63-0.13 d_{w} .
$$

При минимальной величине $d_{w}=1.0$ (прямолинейные или баллистические траектории блуждания наночастиц ОГ) значение $D_{2}$ достигает своей максимальной величины $\sim 2.50$, то есть размерности кластера Виттена-Сандера [13]. При больших значениях $d_{w}>10$ агрегация пластин ОГ за реальное время переработки материалов не реализуется вообще в силу очень большого времени ожидания контакта указанных пластин $[20,21]$.

И в заключение рассмотрим трактовку влияния структуры агрегатов нанонаполнителя, характеризуемой их размерностью $D_{2}$, на свойства нанокомпозитов ПВС/ОГ на примере их модуля упругости $E_{n}$, выполненную в рамках перколяционной модели усиления [22]. Основным уравнением этой модели является следующее соотношение [22]:

$$
\frac{E_{n}}{E_{m}}=1+11\left(\varphi_{n}\right)^{a}
$$

где $E_{m}-$ модуль упругости матричного полимера (отношение $E_{n} / E_{m}$ принято называть степенью усиления на- 


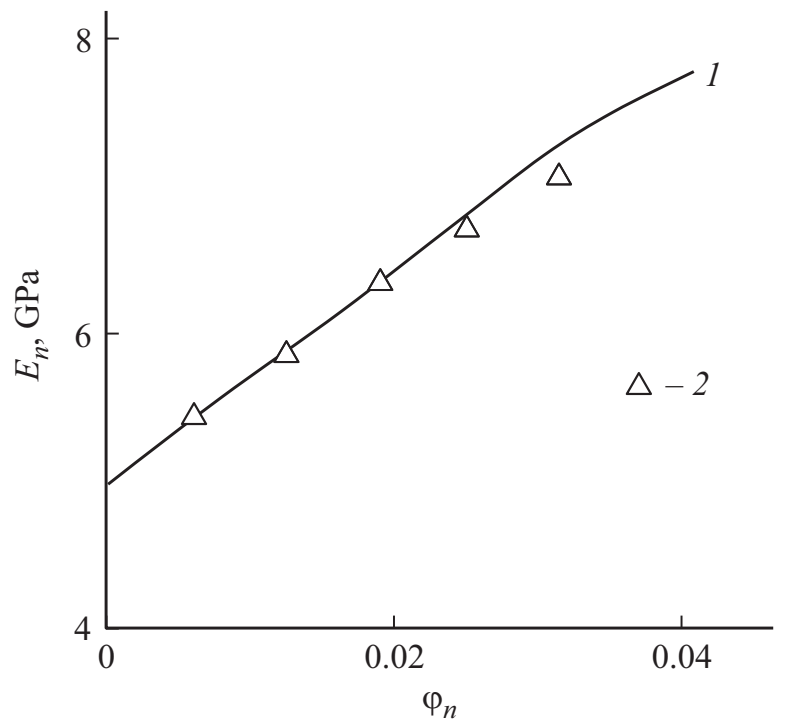

Рис. 4. Сравнение рассчитанной согласно уравнениям (11) и (12) (1) и полученной экспериментально (2) зависимостей модуля упругости $E_{n}$ от объемного содержания нанонаполнителя $\varphi_{n}$ для нанокомпозитов ПВС/ОГ.

нокомпозита), $a$ - перколяционный индекс, связанный с размерностью $D_{2}$ следующим простым уравнением [23]:

$$
a=t-0.25 D_{2}^{2},
$$

где $t-$ стандартный критический перколяционный индекс, равный 1.6 [24].

На рис. 4 приведено сравнение рассчитанной согласно перколяционной модели усиления (уравнения (11) и (12)) и полученной экспериментально зависимостей $E_{n}\left(\varphi_{n}\right)$ при $E_{m}=2.1 \mathrm{GPa}[12]$ для нанокомпозитов ПВС/ОГ, из которого следует хорошее соответствие теории и эксперимента (их среднее расхождение составляет $\sim 12 \%)$. Отметим, что экспериментальная погрешность определения $E_{n}$ для указанных нанокомпозитов равна $\pm 7.3 \%$ [12], т. е. она примерно такая же, как и средняя погрешность теоретической оценки величины $E_{n}$.

\section{3. Выводы}

Таким образом, результаты настоящей работы продемонстрировали, что кластеры (тактоиды) 2D-нанонаполнителя в полимерных средах являются фрактальными объектами. Эти кластеры формируются механизмом роста одновременно на множественных местах зародышеобразования, которыми может быть любая исходная наночастица оксида графена. Основным фактором, определяюшим размерность кластеров, является содержание 2D-нанонаполнителя - его увеличение приводит к снижению размерности блуждания наночастиц оксида графена, повышению эффективной связности структуры и уменьшению времени ожидания контакта наночастиц. Снижение размерности блуждания контролирует рост размерности кластеров (тактоидов) 2D-нанонаполнителя, чья структура определяет свойства конечных наноматериалов.

\section{Список литературы}

[1] G.V. Kozlov, A.K. Mikitaev. Structure and Properties of Nanocomposites Polymer/Organoclay. LAP LAMBERT Academic Publishing GmbH and Comp., Saarbrücken (2013). $318 \mathrm{p}$.

[2] H. Kim, A.A. Abdala, C.W. Macosko. Macromolecules 43, 6515 (2010).

[3] S.H. Shim, K.T. Kim, J.U. Lee, W.H. Jo. Appl. Mater. Interfaces 4, 4184 (2012).

[4] U. Khan, P. May, A. O'Neill, A.P. Bell, E. Boussac, A. Martin, J. Semple, J.N. Coleman. Nanoscale 5, 581 (2013).

[5] U. Khan, P. May, A. O’Neill, J.N. Coleman. Carbon 48, 4035 (2010).

[6] Г.В. Козлов, И.В. Долбин. Прикладная физика 3, 96 (2017).

[7] Х. Стенли. В кн.: Фракталы в физике / Под ред. Л. Пьетронеро, Э. Тозатти. Мир, М. (1988). С. 463.

[8] А.К. Микитаев, Г.В. Козлов, Г.Е. Заиков. Полимерные нанокомпозиты: многообразие структурных форм и приложений. Наука, М. (2009). 278 с.

[9] N. Sheng, M.C. Boyce, D.M. Parks, G.C. Rutledge, J.I. Abes, R.E. Cohen. Polymer 45, 487 (2004).

[10] D.W. Schaefer, R.S. Justice. Macromolecules 40, 8501 (2007).

[11] D.W. Schaefer, J. Zhao, H. Dowty, M. Alexander, E.B. Orler. Soft Mater. 4, 2071 (2008).

[12] Y. Xu, W. Hong, H. Bai, Ch. Li, G. Shi. Carbon 47, 3538 (2009).

[13] H.G.E. Hentschel, J.M. Deutch. Phys. Rev. A 29, 1609 (1984).

[14] Г.В. Козлов, Д.С. Сандитов. Ангармонические эффекты и физико- механические свойства полимеров. Наука, Новосибирск (1994). 261 с.

[15] А.К. Микитаев, Г.В. Козлов. Докл. АН 462, 41 (2015).

[16] T.A. Witten, P. Meakin. Phys. Rev. B 28, 5632 (1983).

[17] W.D. Brown, R.C. Ball. J. Phys. A 17, L517 (1985).

[18] G.V. Kozlov, A.K. Mikitaev, G.E. Zaikov. The Fractal Physics of Polymer Synthesis. Apple Academic Press, Toronto, New Jersey (2014). $359 \mathrm{p}$.

[19] M. Foygel, R.D. Morris, D. Anez, S. French, V.L. Sobolev. Phys. Rev. B 71, 104201 (2005).

[20] R. Jan, P. May, A.P. Bell, A. Habib, U. Khan, J.N. Coleman. Nanoscale 6, 4889 (2014).

[21] H.R. Dennis, D.L. Hunter, D. Chang, S. Kim, J.L. White, J.W. Cho, D.R. Paul. Polymer 42, 9513 (2001).

[22] А.К. Микитаев, Г.В. Козлов. ФТТ 57, 961 (2015).

[23] А.К. Микитаев, Г.В. Козлов. ЖТФ 86, 99 (2016).

[24] И.М. Соколов. УФН 151, 221 (1986).

Редактор К.В. Емцев 